\title{
Mycobacterium fortuitum-induced ER- Mitochondrial calcium dynamics promotes calpain/caspase-12/caspase-9 mediated apoptosis in fish macrophages
}

\author{
Debika Datta ${ }^{1}$, Preeti Khatri ${ }^{1}$, Ambika Singh ${ }^{2}$, Dhira Rani Saha ${ }^{3}$, Gaurav Verma ${ }^{4}$, Rajagopal Raman ${ }^{2}$ and
} Shibnath Mazumder ${ }^{1}$

\begin{abstract}
Mycobacterium fortuitum is a natural fish pathogen. It induces apoptosis in headkidney macrophages (HKM) of catfish, Clarias sp though the mechanism remains largely unknown. We observed $M$. fortuitum triggers calcium $\left(\mathrm{Ca}^{2+}\right)$ insult in the sub-cellular compartments which elicits pro-apototic ER-stress factor CHOP. Alleviating ER-stress inhibited CHOP and attenuated HKM apoptosis implicating ER-stress in the pathogenesis of M. fortuitum. ER-stress promoted calpain activation and silencing the protease inhibited caspase-12 activation. The study documents the primal role of calpain/ caspase-12 axis on caspase- 9 activation in $\mathrm{M}$. fortuitum-pathogenesis. Mobilization of $\mathrm{Ca}^{2+}$ from ER to mitochondria led to increased mitochondrial $\mathrm{Ca}^{2+}\left(\mathrm{Ca}^{2+}\right)_{\mathrm{m}}$ load, mitochondrial permeability transition (MPT) pore opening, altered mitochondrial membrane potential $(\Delta \psi \mathrm{m})$ and cytochrome $c$ release eventually activating the caspase-9/-3 cascade. Ultra-structural studies revealed close apposition of ER and mitochondria and pre-treatment with $\left(\mathrm{Ca}^{2+}\right)_{\mathrm{m}}$-uniporter (MUP) blocker ruthenium red, reduced $\mathrm{Ca}^{2+}$ overload suggesting $\left(\mathrm{Ca}^{2+}\right)_{\mathrm{m}}$ fluxes are MUP-driven and the ERmitochondria tethering orchestrates the process. This is the first report implicating role of sub-cellular $\mathrm{Ca}^{2+}$ in the pathogenesis of $M$. fortuitum. We summarize, the dynamics of $\mathrm{Ca}^{2+}$ in sub-cellular compartments incites ER-stress and mitochondrial dysfunction, leading to activation of pro-apoptotic calpain/caspase-12/caspase-9 axis in $M$. fortuituminfected HKM.
\end{abstract}

\section{Introduction}

$M$. fortuitum is a rapidly growing, atypical, nontubercular mycobacteria affecting wide range of animals including humans ${ }^{1-3}$. In fish, it is one of the etiologic agents causing piscine-tuberculosis or mycobacteriosis, a fatal disease characterized by the development of graywhite nodular structures and presence of single or multiple granulomatous lesions on several parenchymal

\footnotetext{
Correspondence: Shibnath Mazumder (shibnath1@yahoo.co.in) 'Department of Zoology, Immunobiology Laboratory, University of Delhi, Delhi, India

2Department of Zoology, Gut Biology Laboratory, University of Delhi, Delhi, India

Full list of author information is available at the end of the article Edited by A. Rufini
}

organs ${ }^{1}$. Despite its diverse host trophism and zoonotic importance, our knowledge on pathogenic mechanisms and virulence factors expressed by $M$. fortuitum is incomplete.

Alterations in cytosolic calcium $\left(\mathrm{Ca}^{2+}\right)_{\mathrm{c}}$ levels play crucial role in microbial pathogenesis and disease outcome with reports suggesting pro-and anti-apoptotic roles of $\mathrm{Ca}^{2+}$ on mycobacteria-infected macrophages ${ }^{4,5}$. Once $\mathrm{Ca}^{2+}$ is mobilized, it either interacts with various $\mathrm{Ca}^{2+}$ binding proteins or gets sequestered into the $\mathrm{ER}^{6}$. Calcium influx or depletion from the ER induces ERstress $^{6,7}$. The ability to mount ER-stress response is critical for cell survival, but chronic or unresolved ER stress can lead to expression of pro-apoptotic C/EBP 
homologous protein $(\mathrm{CHOP})^{8}$. Though prolonged ERstress has been linked to mycobacterial pathogenesis ${ }^{9-14}$, it has not been reported in $M$. fortuitum.

To mitigate stress, the ER releases $\mathrm{Ca}^{2+}\left(\left(\mathrm{Ca}^{2+}\right)_{\mathrm{ER}}\right)$ through ER-membrane resident inositol-1,4,5-trisphosphate receptors $\left(\mathrm{IP}_{3} \mathrm{R}\right)$ and ryanodine receptors $(\mathrm{RYRs})^{15}$. The $\left(\mathrm{Ca}^{2+}\right)_{\mathrm{ER}}$ is either pumped out of the cell through specific channels or taken up by mitochondria through specific uniport transporter like M1CU1 and VDAc, the latter being facilitated by the known proximity between the two organelles ${ }^{16,17}$.

Calcium overload to mitochondria leads to mitochondrial structure-function alterations eventually releasing the pro-apoptotic cytochrome $c$ to the cytosol ${ }^{17}$. Activation of caspases, a family of cysteine-dependent aspartatedirected proteases, is central to apoptosis and caspase- 12 appears to be the prime caspase involved in ER-stress induced apoptosis ${ }^{18}$. Calpains are $\mathrm{Ca}^{2+}$-activated nonlysosomal cysteine proteases which exist in two isoforms, calpain-1 and calpain- $2^{19}$. Each calpain consists of an 80 $\mathrm{kDa}$ catalytic subunit and a common $28 \mathrm{kDa}$ subunit ${ }^{19}$. The role for calpain in promoting mycobacteria-induced apoptosis is still under investigation ${ }^{10,11,20}{ }^{20}$. Several reports suggested the role of calpains in the activation of caspase- $12^{21,22}$ implicating the plurality of $\mathrm{Ca}^{2+}$ involvement in apoptosis.

The fish immune system is well-developed and comprised of both innate and adaptive immunity. However, unlike other vertebrates, the head kidney (HK) represents the main immunocompetent organ and HKM are important constituents of fish innate immunity ${ }^{23}$. We recently demonstrated the role of caspase- 8 in $M$. fortuitum infection induced HKM apoptosis ${ }^{24}$. However, the interaction of caspase-12 and caspase- 9 is not reported in $M$. fortuitum pathogenesis. In the present study we investigated the the role of caspase- 12 and caspase- 9 in $M$. fortuitum pathogenesis. Our results for the first time implicate $\mathrm{Ca}^{2+}$ dynamics between ER and mitochondria important for $M$. fortuitum induced apoptosis. We suggest that ER-stress espouses apoptosis of $M$. fortuituminfected HKM and activation of calpain/caspase-12/caspase- 9 axis crucial for initiating the apoptotic cascade.

\section{Results}

\section{M. fortuitum-induced intracellular $\mathrm{Ca}^{2+}$ imbalance lead to CHOP- mediated HKM apoptosis}

Previously, we reported that the imbalance in $\left(\mathrm{Ca}^{2+}\right)_{\mathrm{c}}$ triggers apoptosis in $M$. fortuitum-infected fish macrophages $^{24}$. Here, we studied the dynamics of $\left(\mathrm{Ca}^{2+}\right)_{\mathrm{c}}$ in the two sub-cellular compartments, ER and mitochondria.

ER is the main storehouse of intracellular $\mathrm{Ca}^{2+}$ and under stressed condition $\left(\mathrm{Ca}^{2+}\right)_{\mathrm{ER}}$ is released through $\mathrm{IP}_{3} \mathrm{R}$ and RYRs located on the ER-membrane. CHOP is a marker for ER-stress ${ }^{6,7}$ and our preliminary results suggested significant CHOP mRNA expression at $2 \mathrm{~h}$ (Fig. 1b) and protein at $24 \mathrm{~h}$ (data not shown) in M. fortuitum-infected HKM. The HKM were pre-treated with 2-APB and Dant, specific inhibitors for $\mathrm{IP}_{3} \mathrm{R}$ and $\mathrm{RYR}$ respectively ${ }^{25}$, infected with $M$. fortuitum and the changes in CHOP expression and apoptosis studied at $24 \mathrm{~h}$ p.i. We observed decreased expression of CHOP (Fig. 1a) and HKM apoptosis (Figure S1) which suggested positive corelation between $\left(\mathrm{Ca}^{2+}\right)_{\mathrm{ER}}$ depletion and CHOP expression in M. fortuitum infected HKM. In the same line, we observed declined expression of CHOP in presence of intracellular $\mathrm{Ca}^{2+}$ chealator BAPTA/AM (Fig. 1a).

Pre-treatment of HKM with general ER-stress inhibitor 4-PBA down-regulated CHOP expression (Fig. 1a), attenuated caspase- 3 activity and HKM apoptosis (Figure S1). These findings were confirmed using CHOP-siRNA. Transfection with CHOP-siRNA down-regulated CHOP expression at mRNA (Fig. 1c) and protein level (Fig. 1a) besides attenuating $M$. fortuitum-induced HKM apoptosis (Figure S1). Our results for the first time implicated ER-stress induced CHOP in M. fortuitum-induced apoptosis and corroborate with earlier studies suggesting the pro-apoptotic role of $\mathrm{CHOP}$ in mycobacterial pathogenesis.

\section{Mobilization of $\left(\mathrm{Ca}^{2+}\right)_{\mathrm{ER}}$ into mitochondria led to mitochondrial dysfunction}

Mitochondrial dysfunction due to $\mathrm{Ca}^{2+}$ overload is keystone in determining the fate of mycobacteria-infected macrophages ${ }^{26,27}$. HKM were stained with Rhod-2/AM and Mito-Tracker Green FM and observed under the confocal microscope. The increase in Rhod-2 AM fluroscence clearly indicates increased $\left(\mathrm{Ca}^{2+}\right)_{\mathrm{m}}$ uptake following $1 \mathrm{~h}$ of adding the bacteria with peak fluroscence recorded at $6 \mathrm{~h}$ p.i (Figure S2). Calcium influx to the mitochondria occurs through MUP ${ }^{16}$ and to explore this HKM were pre-treated with MUP blocker ruthenium red (RR) and $\left(\mathrm{Ca}^{2+}\right)_{\mathrm{m}}$ dynamics monitored at $6 \mathrm{~h}$ p.i. We observed Rhod-2/AM fluroscence intensity was reversed in presence of RR (Fig. 2a) suggesting $\left(\mathrm{Ca}^{2+}\right)_{\mathrm{m}}$ influx in $M$. fortuitum-infected HKM is uniporter driven.

To investigate the mobilization of $\left(\mathrm{Ca}^{2+}\right)_{\mathrm{ER}}$ to mitochondria HKM were pre-treated separately with Dant, 2APB and $\left(\mathrm{Ca}^{2+}\right)_{\mathrm{m}}$ uptake monitored. The decrease in Rhod-2/AM fluorescence in presence of Dant and 2-APB (Fig. 2a), clearly proved the mobility of $\mathrm{Ca}^{2+}$ from ER to mitochondria. We reasoned, for the uptake of $\left(\mathrm{Ca}^{2+}\right)_{\mathrm{ER}}$ through MUP, the two organelles ought to come in close proximity thereby facilitating the process. $M$. fortuituminfected HKM were examined by TEM at $6 \mathrm{~h}$ and $24 \mathrm{~h}$ p.i. (Fig. 2b) and we observed spatial change in the subcellular organization with mitochondria in close apposition with ER, which appeared more evident in the HKM collected at 24 h p.i. (Fig. 2B3,B4). 
A

HKM HKM+4-PBA

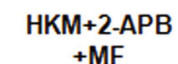

HKM+Dant

HKM+BAPTA/AM
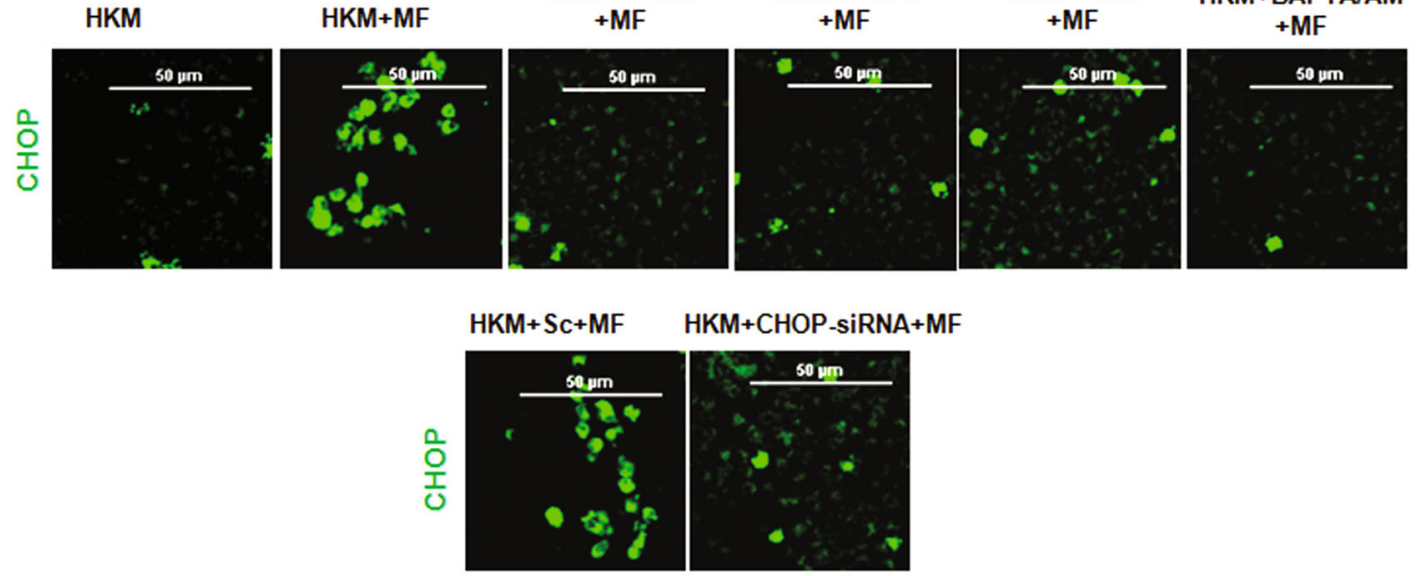

B

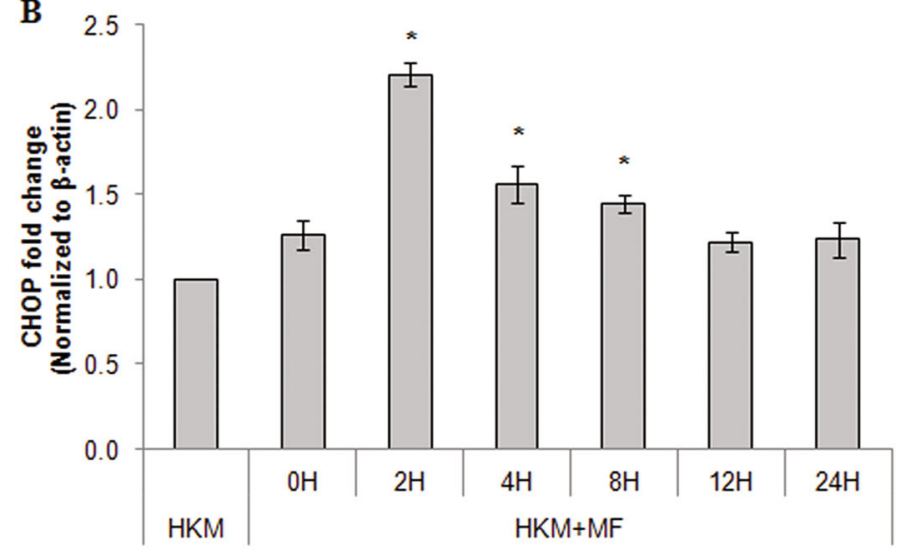

C

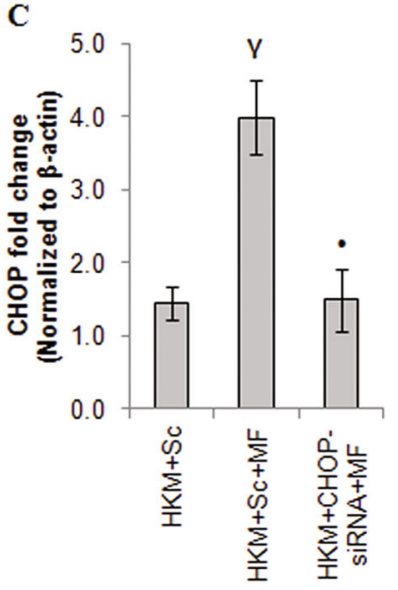

Fig. 1 M. fortuitum induces CHOP- mediated HKM apoptosis. a HKM pre-treated or transfected with indicated inhibitors or siRNAs respectively prior to the infection with M. fortuitum and the CHOP protein expression was studied by confocal microscope using FITC-conjugated secondary antibody. The images are representative of three independent experiments and observed under confocal microscope ( $\times 40)$. b HKM were infected with $M$. fortuitum and CHOP mRNA expression was quantified by qPCR at indicated time p.i. c HKM were transfected with CHOP-siRNA or scrambled siRNA prior to infection with M. fortuitum and CHOP mRNA expression was quantified. Vertical bars represent mean $\pm \mathrm{SE}(n=3) .{ }^{*} P<0.05$, compared to $\mathrm{HKM}^{\mathrm{Y} P}<0.05$, compared to HKM + Sc; ${ }^{*} P<0.05$, compared to HKM + MF + Sc. HKM, control headkidney macrophage; HKM + MF, HKM infected with $M$. fortuitum; HKM + Sc + MF, HKM transfected with scrambled siRNA followed by M. fortuitum infection; HKM + CHOP-siRNA + MF, HKM transfected with CHOP-siRNA infected with M. fortuitum; HKM + 4-PBA + MF, HKM + 2-APB + MF, HKM + Dant + MF, HKM + BAPTA/AM + MF, HKM pre-treated with 4-PBA, 2-APB, Dant, BAPTA/AM respectively followed by $M$. fortuitum infection

The elevation in $\left(\mathrm{Ca}^{2+}\right)_{\mathrm{m}}$ reduces mitochondrial membrane potential $(\Delta \Psi \mathrm{m})^{28}$. We monitored the changes in $\Delta \Psi \mathrm{m}$ in $M$. fortuitum-infected HKM at different time points using the JC-1 dye. The increase in green fluorescence $^{29}$ indicated time-dependent reduction in $\Delta \Psi \mathrm{m}$ in infected HKM (Figure S3). Pre-treatment of HKM with specific inhibitors RR, Dant and 2-APB restored $\Delta \Psi \mathrm{m}$ (Fig. 3a), which confirmed the uptake of $\left(\mathrm{Ca}^{2+}\right)_{\mathrm{ER}}$ on mitochondrial dysfunction. The loss in $\Delta \Psi \mathrm{m}$ leads to the formation of $\mathrm{MPT}^{28}$. The cell-permeant, green-fluorescent, lipophilic dye $\mathrm{DiOC}_{6}$ accumulates in mitochondria and its release is a reliable indicator for $\Delta \Psi \mathrm{m}$ loss and MPT pore opening ${ }^{30}$. When we compared $\mathrm{DiOC}_{6}$ fluoroscence levels in uninfected and M. fortuituminfected HKM, significant loss in fluoroscence levels was noted in the infected HKM (Fig. 3b) which suggested $M$. fortuitum infection leads to loss in $\Delta \Psi \mathrm{m}$ and MPT formation. Among several molecules released via MPT, proapoptotic cytochrome $c$ is important. Hence, the next step was studying cytochrome $c$ release in $M$. fortuituminfected HKM. Our confocal microscopy images suggests the transloction of cytochrome $c$ to the cytosol of $M$. fortuitum-infected HKM (Fig. 3c). Pre-treatment with MPT inhibitor CsA restored $\Delta \Psi \mathrm{m}$ (Fig. 3a), retained $\mathrm{DiOC}_{6}$ (data not shown) and inhibited cytochrome $c$ release (Fig. 3c) in infected HKM. To this we concluded 


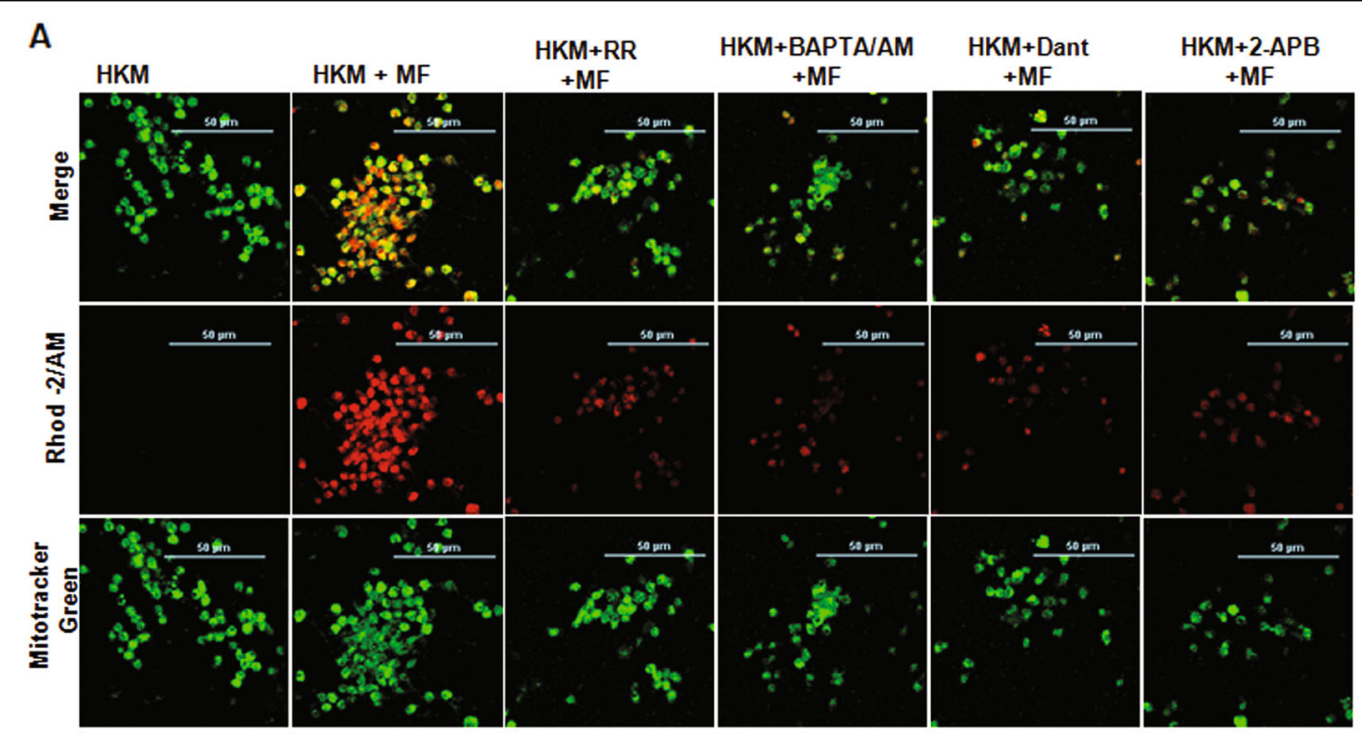

B

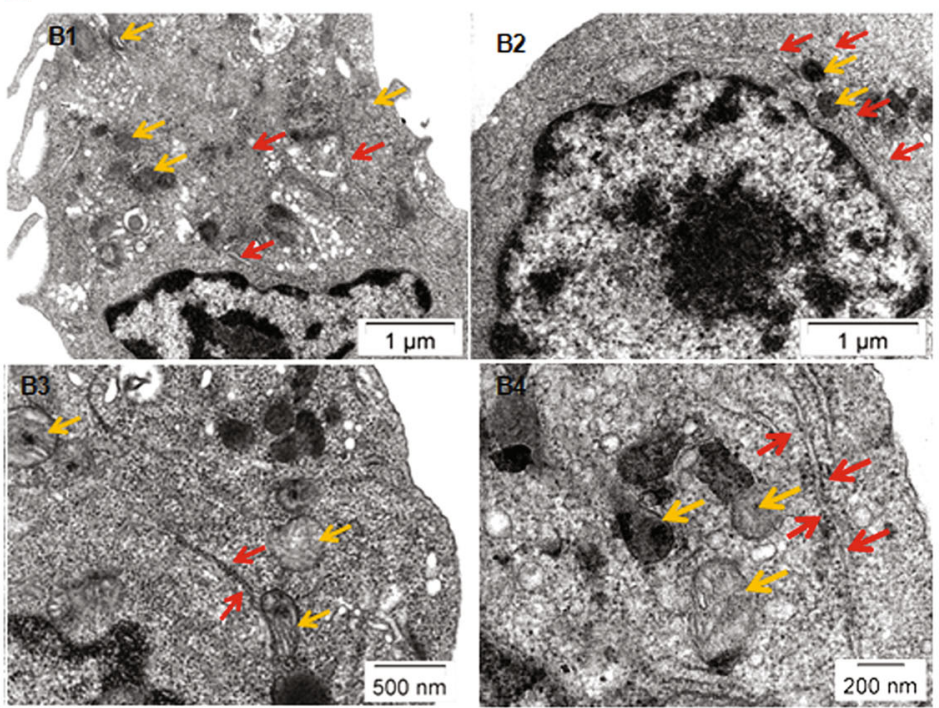

Fig. 2 Close apposition of ER and mitochondria leads to mitochondrial- $\mathrm{Ca}^{2+}$ overload in $\mathbf{M}$. fortuitum infected HKM. a HKM pre-treated with or without indicated inhibitors were infected with $M$. fortuitum and mitochondrial-Ca ${ }^{2+}$ uptake studied $6 \mathrm{~h}$ p.i. by Rhod-2/AM and Mitotracker green marker. The images are representative of three independent experiments and observed under confocal microscope $(\times 40)$. $\mathbf{b}$ Transmission electron microscopy of uninfected HKM (B1), M. fortuitum infected HKM at $6 \mathrm{~h}$ p.i. (B2) and $24 \mathrm{~h}$ p.i. (B3, B4). The images are representative of three independent experiments. HKM, control headkidney macrophage; HKM + MF, HKM infected with M. fortuitum; HKM + RR + MF, HKM + BAPTA/AM $+M F, H K M+$ Dant + MF, HKM + 2-APB + MF, HKM pre-treated with RR, BAPTA/AM, Dant and 2-APB respectively and infected with M. fortuitum. Yellow arrow, mitochondrion; Red arrow, ER

that acquisition of $\left(\mathrm{Ca}^{2+}\right)_{\mathrm{ER}}$ impairs mitochondrial functioning triggering the apoptosis of $M$. fortuitum-infected HKM.

\section{Cytosolic $\mathrm{Ca}^{2+}$ imbalance activates the calpain/caspase-12} axis

Calpains are implicated in apoptosis induced by several mycobacteria $^{11,20}$. In absence of earlier reports, we studied the role of calpain in M. fortuitum-induced HKM apoptosis. Realtime primers for the common $28-\mathrm{kDa}$ regulatory subunit gene (CAPNS1) were designed and qPCR results demonstrated maximum CAPNS1-mRNA expression at $1 \mathrm{~h}$ p.i. (Fig. 4a). We followed this by measuring calpain activity using specific kit and noted maximum calpain activity at $2 \mathrm{~h}$ p.i. (Fig. $4 \mathrm{c}$ ) and selected these two time points for subsequent studies.

Pre-treatment with BAPTA/AM attenuated calpain activity (Fig. 4d) indicating calpain activation to be 

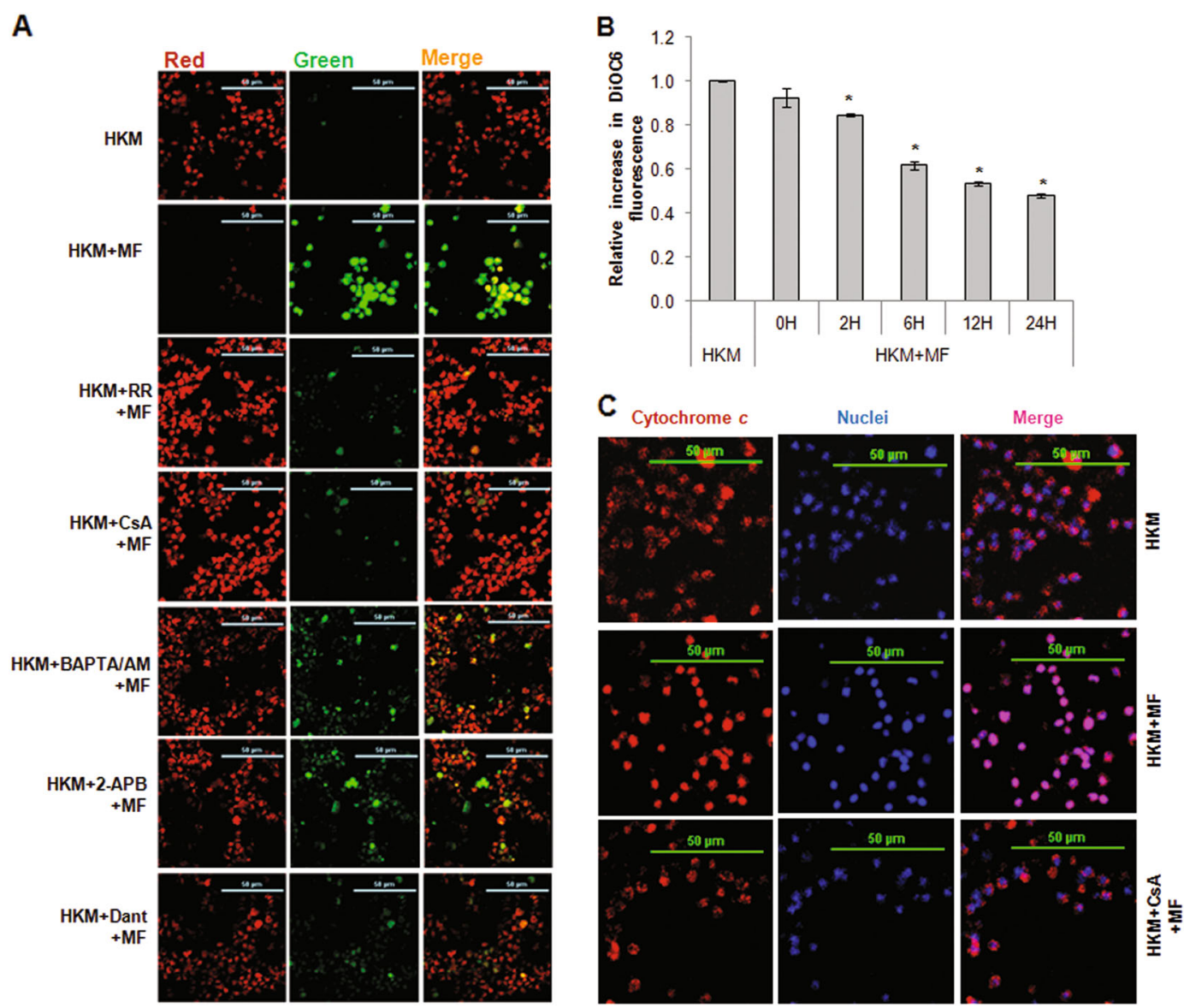

Fig. 3 Alteration in cytosolic $\mathrm{Ca}^{2+}$ homeostasis induces mitochondrial dysfunction triggering $M$. fortuitum-induced HKM apoptosis. a The alteration in $\Delta \psi_{\mathrm{m}}$ was studied using JC-1 dye. HKM pre-treated with or without indicated inhibitors were infected with $M$. fortuitum and at $12 \mathrm{~h}$ p.i. $\Delta \psi_{\mathrm{m}}$ studied by confocal microscopy $(\times 40)$. b MPT formation was studied using DiOC 6 dye. HKM were infected with M. fortuitum for indicated time period and the relative fluorescence intensity of $\mathrm{DiOC}_{6}$ plotted. c HKM pre-treated with or without indicated inhibitors were infected with $M$. fortuitum and the cytosolic translocation of cytochrome $c$ studied by immunoflurescence using TRITC- tagged secondary antibody at $24 \mathrm{~h}$ p.i. The nuclei were stained with DAPI. The images are representative of three independent experiments and observed under confocal microscope $(\times 40)$. Vertical bars represent mean $\pm \mathrm{SE}(n=3) .^{*} P<0.05$, compared to HKM. HKM, control head kidney macrophage; HKM + MF, HKM infected with $M$. fortuitum; HKM + RR + MF, HKM + CSA + MF, HKM + BAPTA/AM + MF, HKM + Dant + MF, HKM + 2-APB + MF, HKM pre-treated with RR, CSA, BAPTA/AM, Dant, 2-APB respectively and infected with $M$. fortuitum

$\mathrm{Ca}^{2+}$-dependent in $M$. fortuitum-infected HKM. Transfection studies were carried out with CAPNS1siRNA and the results from RNAi studies demonstrated significant reduction in $M$. fortuitum induced calpainmRNA expression (Fig. 4b), -protein activity (Fig. 4d) and HKM apoptosis (Figure S1). HKM were pre-treated with pan-calpain inhibitor PD150606 and the changes in calpain and caspase-3 activity and HKM apoptosis studied. We observed significant attenuation in calpain activity (Fig. 4d), caspase-3 activity and HKM apoptosis (Figure S1) in presence of PD150606. The inactive analog PD145305 had no effect on calpain and caspase3 activation as well as HKM apoptosis (data not shown). Calpains exists in two isoforms and our interest was to identify their relative involvements in
M. fortuitum-induced HKM apoptosis. In this direction, HKM pre-treated separately with calpain $1 i$ and calpain $2 i$ were infected with $M$. fortuitum and caspase3 activation and apoptosis monitored. We observed calpain $1 i$ and calpain $2 i$ were equally effective in inhibiting calpain activation (Fig. 4d), caspase-3 activation and HKM apoptosis (Figure S1) suggesting both calpain isoforms contribute equivalently to $M$. fortuitum-pathogenesis.

ER-stress can induce the activation of calpains ${ }^{31}$. To correlate ER-stress with calpain activity the HKM were pre-treated with 4-PBA and calpain activity studied in the infected cells. We observed that 4-PBA pre-treatment led to significant reduction in calpain activiation (Fig. 4d) in M. fortuitum-infected HKM. These findings suggested 

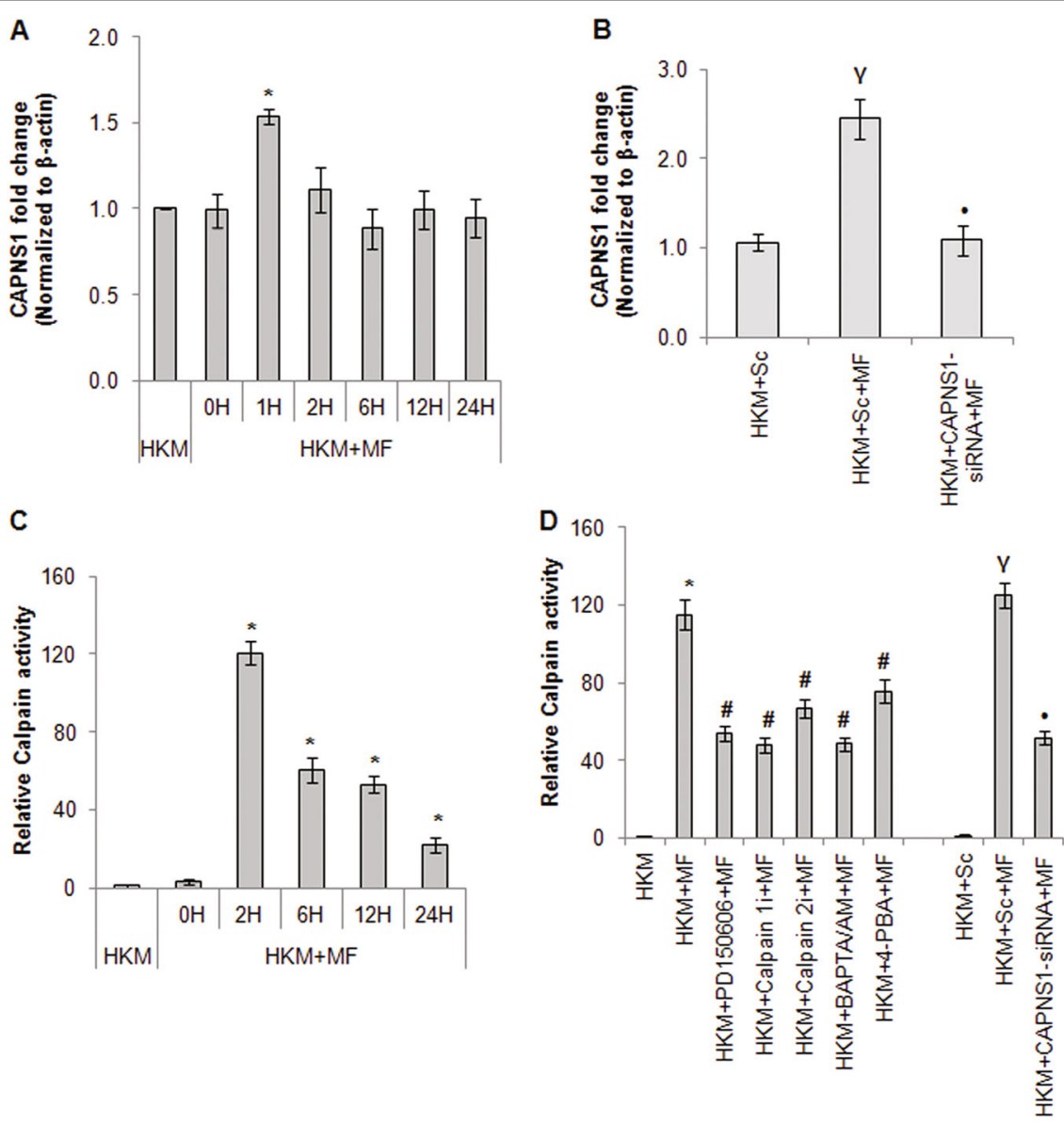

E

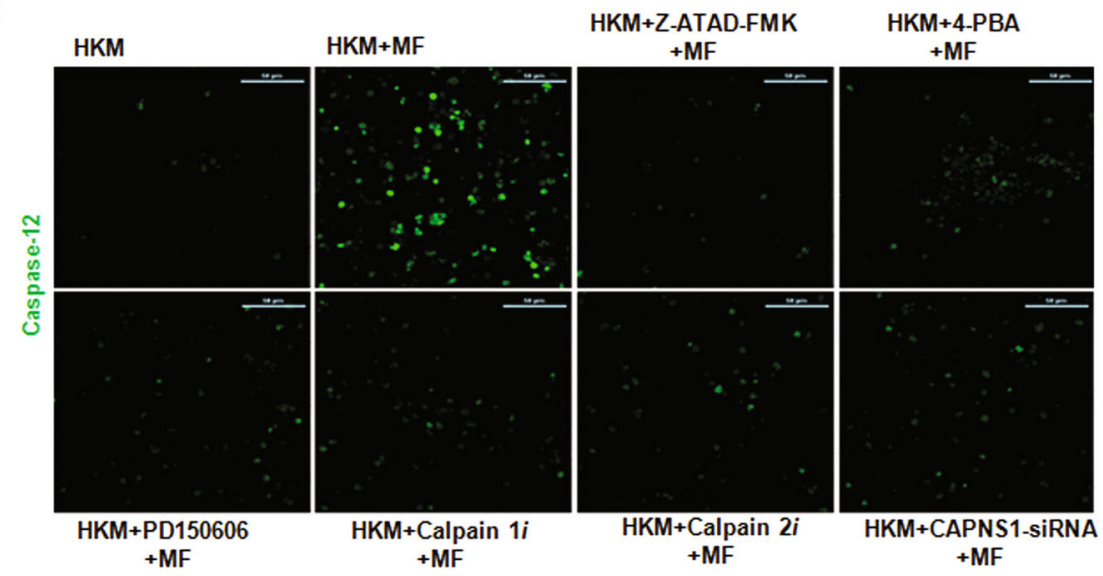

Fig. 4 Activation of calpain/caspase-12 axis is consequent to ER-stress in $\mathbf{M}$. fortuitum infected HKM. a HKM were infected with M. fortuitum and at indicated time p.i. CAPNS1-mRNA expression was quantified by qPCR. $\mathbf{b}$ HKM were transfected separately with CAPNS1-siRNA or scrambled siRNA and CAPNS1-mRNA expression was quantified by qPCR. c HKM were infected with M. fortuitum and at indicated time p.i. calpain activity was measured using assay kits. $\mathbf{d}$ HKM transfected separately with CAPNS1-siRNA, scrambled siRNA or pre-treated with indicated inhibitors were infected with $M$. fortuitum and calapin activity measured at $2 \mathrm{~h}$ p.i. e HKM pre-treated with indicated inhibitors or transfected with CAPNS1-siRNA were infected with $M$. fortuitum and caspase-12 activity was studied at $24 \mathrm{~h}$ p.i. using specific kit and observed under confocal microscope $(\times 40)$. The images are representative of three independent experiments. Vertical bars represent mean $\pm \mathrm{SE}(n=3)$. $^{*} P<0.05$, compared to HKM; $\gamma P<0.05$, compared to HKM + Sc; ${ }^{\# P} P<0.05$, compared to HKM + MF; $\cdot P<0.05$, compared to HKM + SC + MF. HKM, control head kidney macrophage; HKM + $\mathrm{Sc}$, HKM transfected with scrambled siRNA; HKM + MF, HKM infected with M. fortuitum; HKM + Sc + MF, HKM transfected with scrambled siRNA followed by M. fortuitum infection; HKM + CAPNS1-siRNA + MF, HKM transfected with CAPNS1-siRNA then infected with M. fortuitum; HKM + PD150606 + MF, HKM + Calpain1i + MF, HKM + Calpain2i + MF, HKM + 4-PBA + MF, HKM + BAPTA/AM + MF, HKM + Z-ATAD-FMK + MF, HKM pretreated with PD150606, Calpain1i, Calpain2i, 4-PBA, BAPTA/AM, Z-ATAD-FMK respectively and infected with M. fortuitum 
ER-stress contribute towards pro-apoptotic calpain activation in $M$. fortuitum infected HKM.

We followed this by studying ER-stress induced caspase-12 activation. HKM were infected with $M$. fortuitum and caspase-12 expression monitored under the confocal microscope at $24 \mathrm{~h}$ p.i., the end point of the study. We observed significant caspase-12 activity in infected HKM. Pre-treatment with caspase-12 inhibitor Z-ATAD-FMK and 4-PBA inhibited caspase-12 activity (Fig. 4e) and attenuated M. fortuitum-induced HKM apoptosis (Figure S1). Thus, we concluded that ER-stress leads to pro-apoptotic caspase-12 activation in $M$. fortuitum-infected HKM.

Earlier studies suggested the involvement of calpains in caspase-12 activation ${ }^{21}$. To investigate this, the HKM were treated with PD150606, Calpain $1 i$, Calpain $2 i$ or transfected with CAPNS1-siRNA prior to $M$. fortuitum infection and caspase- 12 activity studied. We observed the down-regulation in caspase-12 activity in presence of PD150606, Calpain 1i, Calpain $2 i$ and CAPNS1-siRNA respectively (Fig. 4e). PD145305 failed to inhibit caspase12 activation (data not shown), suggesting the role of calpain on caspase-12 activation in M. fortuitum-infected HKM. Based on these findings we concluded that $M$. fortuitum-induced ER-stress lead to the activation of proapoptotic calpain/caspase-12 axis in HKM.

\section{Caspase-12 and cytochrome $c$ instigate caspase-9 activation to expedite $M$. fortuitum induced-HKM apoptosis}

The release of cytochrome c leads to activation of caspase- 9 and cellular apoptosis ${ }^{17}$. We set out to determine the role of cytochrome $c$-caspase- 9 axis in $M$. fortuitum-induced HKM apoptosis. Enhanced caspase-9 activity was noted in M. fortuitum infected HKM (Fig. 5) and pre-treatment with caspase-9 inhibitor Z-LEHDFMK significantly attenuated caspase- 3 activity and HKM apoptosis (Figure S1) implicating the involvement of caspase-9 in $M$. fortuitum pathogenesis. We extended our study and noted that caspase- 9 activity was attenuated in the presence of MPT inhibitor CsA (Fig. 5) which ensured cytochrome $c$ released due to MPT is critical for caspase-9 activation in M. fortuitum-infected HKM.

In an earlier study it was noted that caspase-12 influences caspase- 9 further intensifying the apoptotic cascade $^{32}$. To probe this, we pre-treated the HKM with the caspase-12 inhibitor Z-ATAD-FMK and assayed caspase9 activity. It is evident from Fig. 5 that caspase- 9 activity was attenuated in Z-ATAD-FMK pre-treated HKM. Based on these observations we propose that both cytochrome $c$ and caspase-12 mediated pathways intersect at caspase- 9 to expedite HKM death induced by $M$. fortuitum.

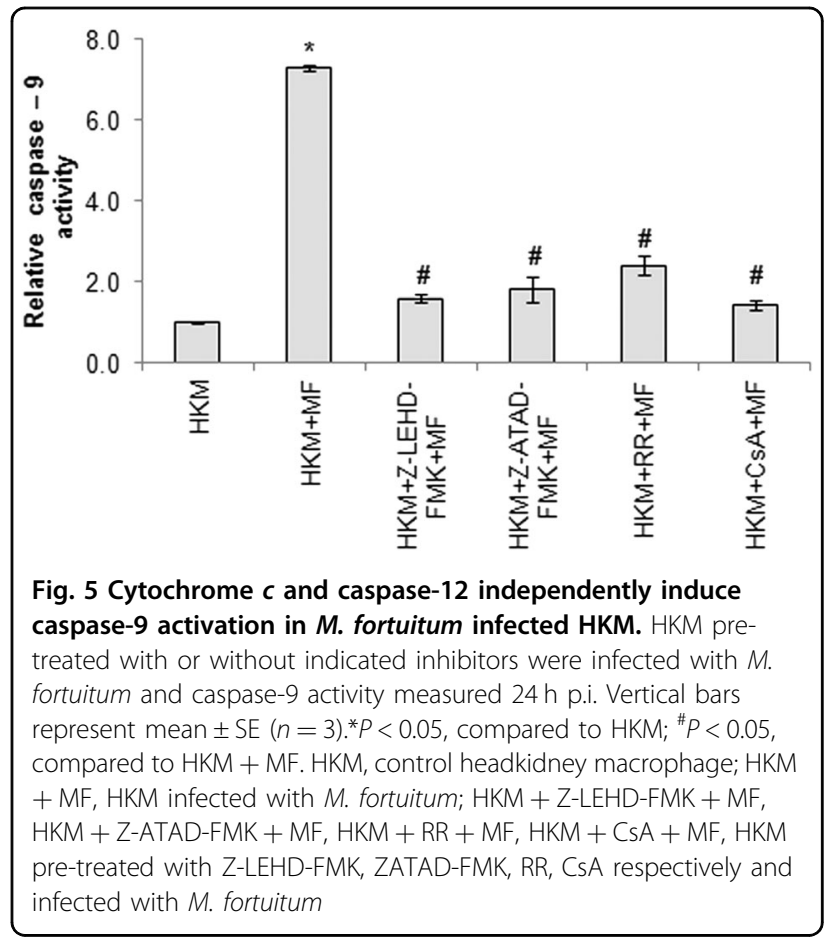

\section{Discussion}

In the present study, we report that the cross-talk between ER and mitochondria aggravates down-stream apoptotic signaling in $M$. fortuitum infected HKM wherein, $\mathrm{Ca}^{2+}$ dynamics in the sub-cellular compartments plays a crucial role on expediting the death program.

The pro-apoptotic transcription factor $\mathrm{CHOP}$ is marker for ER-stress ${ }^{7}$. The expression of CHOP was significantly reduced in presence of BAPTA/AM, an intracellular $\mathrm{Ca}^{2+}$ chelator implicating alteration in intracellular $\mathrm{Ca}^{2+}$ homeostasis is closely related to ER-stress generation in $M$. fortuitum infected macrophages. We hypothesize, elevated cytoslic $\mathrm{Ca}^{2+}$ induces protein misfolding affecting protein loading in ER and BAPTA/AM might reduce misfolded proteins thus attenuating ER-stress induced by M. fortuitum. 4-PBA is low molecular weight chemical chaperone which has several biological effects of which inhibiting ER-stress is important ${ }^{33}$. It helps in stabilizing protein conformation thereby improving the folding capacity of ER and represses UPR ${ }^{33,34}$. We hypothesized 4-PBA would prevent HKM apoptosis through inhibition of ER-stress induced CHOP expression. We observed that alleviating ER-stress with 4-PBA down-regulated $\mathrm{CHOP}$ expression coupled with decline in caspase- 3 activity and HKM apoptosis. Besides, CHOP-siRNA suppressed caspae-3 activity and HKM apoptosis. CHOP has 'versatile role' in ER-stress mediated apoptosis. It downregulates BCL-2 anti-apoptotic proteins at transcriptional level triggering the mitochondrial apoptotic cascade $^{35}$. It has been also reported CHOP induces the 
transcription of ER oxidoreductin $1 \alpha$ (ERO1 $\alpha)$ leading to hypoxic milieu in ER, that enhances downstream death signaling ${ }^{35,}{ }^{36}$. In this context, it would be interesting to study the versatility of CHOP in M. fortuitum induced apoptosis.

Based on these observations, we propose that the ERoverload and unresolved ER-stress induced by $M$. fortuitum is a crucial trigger to induce HKM apoptosis. The role of cytosolic $\mathrm{Ca}^{2+}$ on ER-stress generation and macrophage apoptosis has been reported in several mycobacteria $^{10,11}$ and our results extends this to M. fortuitum, suggesting it to be common virulence trait for different mycobacterial species. This is the first report suggesting that $M$. fortuitum can induce ER-stress with pathological implications in host. Keeping the diverse host trophism of $M$. fortuitum in view it would be interesting to see whether same pathogenic mechanisms are employed by the bacteria to induce pathogenesis across species barrier.

Our results with 2-APB and Dant showed significant suppression in $\mathrm{CHOP}$ expression implicating the definite role of $\left(\mathrm{Ca}^{2+}\right)_{\mathrm{ER}}$ depletion in in M. fortuitum pathogenesis. The next step was to look for the likely down-stream targets induced by $\mathrm{Ca}^{2+}$ and calpain appeared attractive. Calpains are non-lysosomal cysteine proteases consisting of $80-\mathrm{kDa}$ catalytic subunit and a common $28-\mathrm{kDa}$ regulatory subunit, calpain small-1 (CAPNS1), encoded by CAPNS1 gene required for functioning ${ }^{37,38}$. We observed over-expression of CAPNS1 mRNA and higher calpain activity and inhibiting the protease activity resulted in down regulation of caspase- 3 and $M$. fortuitum induced HKM apoptosis. The presence of the two different tissue isoforms, calpain- 1 and -2 is well documented in fish ${ }^{39,40}$. Our results demonstrated that both isoforms are important for inducing $M$. fortuitum induced HKM apoptosis. Alleviating ER-stress with 4-PBA significantly reduced calpain activity suggesting calpain activation consequent to ER-stress generation in $M$. fortuitum infected HKM. Although the involvement of ER-calpain axis has been reported in the pathogensis of both atypical ${ }^{11}$ and typical mycobacteria ${ }^{20}$ our results constitute the first report in $M$. fortuitum suggesting calpain activation as an evolutionary conserved virulence attribute for mycobacteria.

We posited that the efflux of ER-Ca ${ }^{2+}$ into the cytoplasm through $\mathrm{IP}_{3}$ and $\mathrm{RYR}$ activates calpain to initiate downstream effects. Hence, calpain activity was studied in presence of 2-APB and Dant. Importantly, 2-APB and Dant, failed to completely abrogate calpain activity emphasizing $\mathrm{Ca}^{2+}$ efflux in M. fortuitum infected HKM involves multitude of pathways.

The activation of caspase-12 as a marker for ER-stress has recently been demonstrated in fish $^{41}$. It has been observed that calpain cleaves the ER-resident procaspase-12 to active caspase- 12 further intensifying the apoptotic cascade. Our results for the first time showed calpain-induced caspase-12 activation to be an important step in the pathogenesis of $M$. fortuitum. It was suggested that calpain-2 activation leads to cleavage of caspase- $12^{21}$. We did not observe any difference in the ability of either calpain isoforms on caspase-12 activation suggesting caspase- 12 to be substrate for both calpain-1 and -2 in $M$. fortuitum pathogenesis. We are currently studying the mechanisms of calpain dependency on caspase-12 activation in $M$. fortuitum pathogenesis.

An obligatory step in the ER-stress pathway is mitochondrial dysfunctioning with $\mathrm{Ca}^{2+}$ playing an active role on initiating the process ${ }^{16,17}$. The participation of $\mathrm{Ca}^{2+}$ in M. fortuitum induced HKM apoptosis prompted us to explore ER-mitochondrial cross-talk in the pathogenesis induced by the bacterium. We observed overflow of $\left(\mathrm{Ca}^{2}\right.$ $\left.{ }^{+}\right)_{\mathrm{ER}}$ to mitochondria of M. fortuitum infected HKM. Mitochondrial calcium overload alters mitochondrial membrane permeability and leads to opening of MPT. Our results showed that pre-treatment with MPT inhibitor CsA inhibited $\Delta \Psi \mathrm{m}$ dissipation, caspase-3 activation and HKM apoptosis suggesting MPT formation to be associated with $M$. fortuitum pathogenesis. It has been reported that a close physical contact is pre-requisite for the mobility of $\mathrm{Ca}^{2+}$ from ER to mitochondria and MUP are the likely "hotspots" through which $\mathrm{Ca}^{2+}$ enters the mitochondria ${ }^{6}$. Ultra-structural studies depicted close apposition between the two organelles with mitochondria docked onto the ER. We used the specific MUP inhibitor $\mathrm{RR}$ and observed diminished mitochondrial $\mathrm{Ca}^{2+}$ uptake with concomitant decline in caspase- 3 activity and HKM apoptosis. Similar reduction in mitochondrial $\mathrm{Ca}^{2+}$ load and MPT formation were also noted when the HKM were pre-treated with 2-APB and Dant. We propose that the interim association between the two organelles facilitates the efficient transfer of $\left(\mathrm{Ca}^{2+}\right)_{\mathrm{ER}}$ via MUPs leading to mitochondrial dysfunctioning and apoptosis of HKM.

MPT formation leads to overproduction of superoxide anions and release of pro-apoptotic cytochrome $c$ into the cytosol. Increased amount of superoxide has been frequented with apoptosis induced by mycobacterial pathogens $^{10,}{ }^{11}$. Our preliminary results suggested the role of superoxide anions on M. fortuitum induced HKM apoptosis $^{24}$. We hypothesize that MPT formation contributes to the overall process of apoptosis through the release of superoxide anions in the infected HKM. Blocking MPT formation by CsA significantly down-regulated cytochrome $c$ release in cytosol suggesting close association between MPT formation and cytochrome $c$ release in $M$. fortuitum infected HKM. We detected significant caspase9 activity in $M$. fortuitum infected HKM which could be inhibited in presence of CsA suggesting MPT formation and cytochrome $c$ release having a significant role on caspase-9 activation in M. fortuitum infected HKM. Earlier studies suggested a role of caspase-12 on activating 
the caspase-9/-3 axis $^{31,}$ 42. We observed, inhibiting caspase-12 significantly down-regulated the activation of caspase- $9 / 3$ axis, suggesting that caspase- 9 can be activated by multiple pathways in $M$. fortuitum infected HKM. The involvement of caspase-9 is well documented in pathogenesis induced by several mycobacteria ${ }^{12,} 43-45$ our study extends this to $M$. fortuitum.

Mycobacteria-induced cell death depends on several factors including nature of bacterial strains, MOI, host cell types and durations of infection ${ }^{46-48}$. There are also reports suggesting mycobacteria causes caspasedependent apoptotic and caspase-independent necrotic death depending on varied conditions of infection ${ }^{48}$. In this study, we observed caspase mediated apoptosis of catfish macrophages at $24 \mathrm{~h}$ p.i. and caspase- 12 /caspase- 9 axis playing crucial role in triggering the process. The role of caspase-12 in ER-stress induced apoptosis is contentious with studies suggesting caspase- 12 not to be part of UPR-induced apoptosis ${ }^{49}$. There are also reports that ER-stress induces caspase-independent necrosis ${ }^{48}$. In these studies, chemical stressers (tunicamycin, thapsigargin etc) have been used to induce and study the consequences of ER-stress, which may not be akin to pathogen induced stress. Nonetheless, presence of late apoptotic $\left(\mathrm{AV}^{+} \mathrm{PI}^{+}\right)$and necrotic $\left(\mathrm{AV}^{-} \mathrm{PI}^{+}\right)$subsets in $M$. fortuitum infected HKM along with inability of caspase inhibitors to completely abrogate cell death suggests a subset of HKM might be undergoing caspaseindependent death. In this context, it would be interesting to study $\mathrm{CHOP}$ mediated necroptosis or necrosis in the immunopathogenesis of $M$. fortuitum at later time points or with higher MOI.

Cytochrome $c$ also binds to $\mathrm{IP}_{3}$ receptors on the ER facilitating $\left(\mathrm{Ca}^{2+}\right)_{\mathrm{ER}}$ release ${ }^{50}$. We believe that besides its direct involvement in caspase-9 mediated apoptosis cytochrome $c$ also contributes towards mitochondrial $\mathrm{Ca}^{2}$ ${ }^{+}$influx necessary for induction of HKM apoptosis. MPT formation leads to overproduction of superoxide anions and release of pro-apoptotic cytochrome $c$ into the cytososl and increased amount of superoxide anions has been implicated in apoptosis induced by mycobacterial pathogens $^{10,11}$. We hypothesize that MPT formation also contributes to the overall process of apoptosis through the release of superoxide anion in the infected HKM.

The role of mitochondria in piscine mycobacteriosis is not clear. Recently it has been reported in M. marinumzebrafish model that by modulating mitochondrial permeability transition pore formation mycobacteria induced

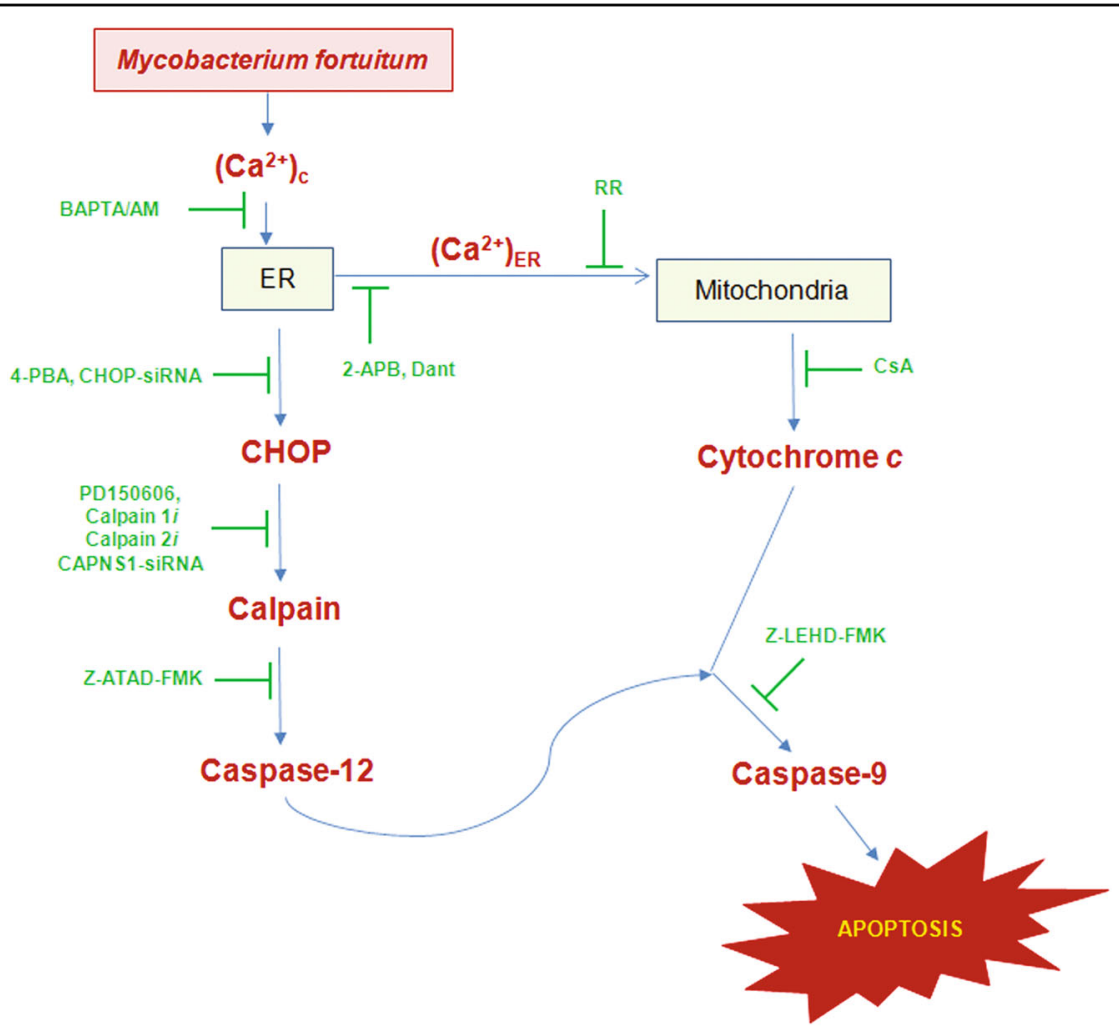

Fig. 6 Overview of the work. M. fortuitum-induced alteration in $\mathrm{Ca}^{2+}$ homeostasis aggravates ER-stress. This leads to (i) activation of the calpain/ caspase-12 axis and (ii) mitochondrial dysfunction resulting in the cytosloic translocation of cytochrome $c$. The two pathways converge at caspase- 9 leading to apoptosis of piscine macrophages 
programmed necrosis (necroptosis) ${ }^{51}$. We believe that these contradictions likely underline the complex and dynamic nature of the mycobacterial pathogenesis.

To conclude, our results established that $\mathrm{Ca}^{2+}$ dynamics of in sub-cellular compartments lead to ER-stress generation and mitochondrial dysfunctioning in $M$. fortuitum-infected HKM. We propose that altered cytosloic$\mathrm{Ca}^{2+}$ triggers ER-stress accompanied with $\left(\mathrm{Ca}^{2+}\right)_{\mathrm{ER}}$ release. $\mathrm{ER}^{-\mathrm{Ca}^{2+}}{ }^{2+}$ besides activating the calapin-caspase12 axis also induces mitochondrial dysfunctioning; the two pathways converge at caspase-9 initiating caspase-3 mediated HKM apoptosis (Fig. 6). These findings would be useful for understanding the pathogenesis of $M$. fortuitum as well as controlling mycobacteriosis.

\section{Materials and methods}

Bacterial strains and growth conditions

Mycobacterium fortuitum (Strain MTCC 993) purchased from Microbial Type Culture Collection and Gene Bank (MTCC), Chandigarh, India were grown at $30^{\circ} \mathrm{C}$ in standard Middlebrook $7 \mathrm{H} 9$ broth (HiMedia). The identity of the isolates was confirmed by AFB staining and $16 \mathrm{~S}$ rDNA sequencing. As the bacteria are sensitive to amikacin $50 \mu \mathrm{g} / \mathrm{mL}$ of the same was added to eradicate the extracellular bacteria ${ }^{24}$.

\section{Isolation of HKM and infection with $M$. fortuitum}

All animal experiments were approved by the Animal Ethics Committee, University of Delhi (DU/ZOOL/IAEC$\mathrm{R} / 2013 / 34$ ) and carried out in accordance with the protocols approved by The Prevention of Cruelty to Animals Act, Govt. of India. The methods for catfish (Clarias sp) maintenance and the protocols for obtaining HKM and infecting them with $M$. fortuitum (multiplicity of infection (MOI 10)) has been described earlier ${ }^{24}$.

\section{Inhibitors used}

Intrcellular $\mathrm{Ca}^{2+}$ chelator (1, 2-Bis (2-aminophenoxy) ethane-N,N, $\mathrm{N}^{\prime}, \mathrm{N}^{\prime}$-tetraacetic acid tetrakis (acetoxymethyl ester), BAPTA/AM, $5 \mu \mathrm{M}$ )), ER-stress alleviator (4-Phenyl butyric acid, 4-PBA, $10 \mu \mathrm{M})$, IP3 receptor antagonist (2Aminoethyl diphenylborinate, 2-APB, $100 \mu \mathrm{M}$ ), calpain 1 inhibitor ( $\mathrm{N}$-acetyl-leucyl-leucyl-norleucinal, Calpain $1 i$, $50 \mu \mathrm{M})$, calpain 2 inhibitor (N-acetyl-leucyl-leucylmethioninal, Calpain $2 i, 50 \mu \mathrm{M})$, mitochondrial uniporter inhibitor (Ruthenium Red, RR, $20 \mu \mathrm{M}$ ), were purchased from Sigma. Pan-calpain inhibitor ([3-(4-iodophenyl)-2mercapto-(Z)-2-propenoic acid], PD150606, $50 \mu \mathrm{M})$, negative control for calpain inhibitor (2-mercapto-3phenypropionic acid, PD145305, $50 \mu \mathrm{M})$, rynodine receptor blocker (Dantrolene, Dant, $20 \mu \mathrm{M}$ ) were purchased from Calbiochem. MPTP blocker (Cyclosporin A, CsA, $5 \mu \mathrm{M}$ ) was from US Biological. Caspase-12 inhibitor (Z-ATAD-FMK, $10 \mu \mathrm{M}$ ) and caspase-9 inhibitor (Z-
LEHD-FMK, $10 \mu \mathrm{M})$ were purchased from Biovision. Cytotoxicity test was done to determine the concentration of inhibitors used (data not shown). The inhibitors were added to the cell culture $1 \mathrm{~h}$ prior to the $M$. fortuitum infection and maintained throughout the experiment. The viability of HKM treated with the indicated concentrations of the inhibitors remained maintained at all-time points as checked by the trypan blue (0.4\%) dye exclusion method. The concentrations of different inhibitors used for the study also had no effect on bacterial growth per se when added to Middlebrook 7H9 or complete-RPMI.

\section{siRNA Transfection}

The siRNA transfection was carried out using HiPerFect Transfection Reagent (Qiagen), as described earlier ${ }^{24,52}$. Transfection efficiency was confirmed by Real-Time PCR, protein and apoptosis assays. Five nano mole each of targeted CHOP [SENSE AUGAAGACUUGCAAGAUAUdTdT \& ANTISENSE AUAUCUUGCAAGUCUUCAUdTdT], CAPNS1 [SENSE CAUGGACUUCGACAACUACdTdT \& ANTISENSE GUAGUUGUCGAAGUCCAUGdTdT], and siRNA Universal negative CONTROL (Sigma) were used for this study.

\section{RNA isolation, CDNA synthesis, cloning, amplification,} sequencing and quantative real-time PCR

HKM $\left(2 \times 10^{7}\right)$ transfected separately with or without targeted or scrambled siRNA were infected with $M$. fortuitum and at indicated time p.i. the total RNA was isolated using TRIZOL (Sigma). cDNA was prepared from 1 $\mu \mathrm{g}$ of DNase treated (RNase-free) RNA using first strand cDNA synthesis kit as per manufacturer's instructions (MBI Fermentas). Degenerate primers were designed using the homologous stretch across fish for CHOP and all vertebrates for the common calpain small sub-unit (CAPNS1) as the template (Table S1). The cDNA was amplified; the amplicons extracted using HiPura gel extraction kit (HiMedia), cloned into pGEM-T EASY vector (Promega) and sequenced (Macrogen). The sequences obtained (Table S2) were aligned to nBLAST and submitted to EMBL or NCBI database. The sequence for CHOP (accession number EMBL-LK054407) showed $80 \%$ identity with CHOP-mRNA sequence of zebrafish (Danio rerio) and the sequence for CAPNS1 (accession number NCBI-KM242108) showed $80 \%$ sequence identity with CAPNS1 sub-unit of Atlantic salmon (Salmo salar).

The quantification of CHOP and CAPNS1 mRNA were performed using SYBR green PCR Master Mix (Applied Biosystems) by Real-Time PCR (ABI ViiA, Applied Biosystems) as described earlier. The gene specific real-time primers for CHOP (FP:5'- GTTGGAGGCGTGGTATGAAG-3'; RP:5'-GAAACTCCGGCTCTTTCTCG-3') 
and CAPNS1 (FP:5'-ACGGGAAAACTGGGGTTCG-3'; RP:5'-TGCTTATAGACAGCCTGCCAC-3') have been used. Expression levels of target genes were analyzed by comparative $\Delta \Delta C_{\mathrm{T}}$ method using $\beta$-actin as the internal control (endogenous control) and uninfected HKM $(0 \mathrm{~h})$ was used as the calibrator ${ }^{24}$.

\section{Apoptosis study}

HKM $\left(1 \times 10^{6}\right)$ transfected or pre-treated with or without indicated concentrations of targeted or scrambled siRNAs or specific inhibitors were infected with or without $M$. fortuitum (MOI 10) and apoptosis studied at $24 \mathrm{~h}$ p.i. by Hoechst 33342 (Sigma) and annexinV-FITC \& propidium iodide (AV-PI, BD-Pharmingen) staining in fluroscence microscope $(\times 40$, Nikon Eclipse 400$)$ as described earlier ${ }^{24}$.

\section{Immunofluorescence studies}

HKM $\left(5 \times 10^{6}\right)$ transfected or pre-treated with or without targeted or scrambled siRNAs and specific inhibitors were infected with or without $M$. fortuitum. At the indicated time p.i. the HKM were washed and fixed in methanol and were incubated in blocking and permeabilizing solution (PBS, $2 \mathrm{mg} / \mathrm{mL}$ BSA, $0.2 \mathrm{mg} / \mathrm{mL}$ saponin) for $1 \mathrm{~h}$ at room temperature. The cells were washed and incubated with primary antibodies; $\mathrm{CHOP}$ (mouse, 1:100, Cell Signalling Technology) and cytochrome $c$ (mouse, 1:100, Biovision) separately overnight at $4{ }^{\circ} \mathrm{C}$. The HKM were washed in PBST (PBS containing $0.1 \%$ Tween-20) and stained with FITC or TRITC conjugated secondary antibodies $(1: 250)$ for $3 \mathrm{~h}$ at $30^{\circ} \mathrm{C}$ and visualized under confocal microscope $(\times 40$ oil immersion, 1.30 NA, Nikon Eclipse A1Rsi-TiE-300 ${ }^{41}$. Nuclei were stained with DAPI $(1 \mu \mathrm{g} / \mathrm{mL})$ before mounting on microslide.

\section{Imaging analysis of $\left(\mathrm{Ca}^{2+}\right)_{\mathrm{m}}$ uptake}

The HKM $\left(2 \times 10^{6}\right)$ pre-treated with or without specific inhibitors were infected with or without $M$. fortuitum. At indicated time p.i. the cells were washed, loaded simultaneously with Rhod-2/AM and mitotracker green (50 $\mathrm{nM}$, Molecular Probes), incubated at $30^{\circ} \mathrm{C}$ for $30 \mathrm{~min}$ then washed, mounted on microslide with cover slips using fluoroshield and visualized under confocal microscope $(\times 40$ oil immersion, 1.30 NA, Nikon Eclipse A1Rsi-TiE-300).

\section{Measurement of $\Delta \Psi_{m}$}

The changes in $\Delta \Psi \mathrm{m}$ and the induction of the mitochondrial permeability transition (MPT) were studied using JC1 (Cayman) and $\mathrm{DiOC}_{6}$ (Sigma) dyes respectively. In case of JC-1, cells with a high $\Delta \Psi \mathrm{m}$ were those forming J-aggregates and in case of $\mathrm{DiOC}_{6}$, high $\Delta \Psi \mathrm{m}$ was attributed to cells with a high fluorescence signal.
HKM $\left(2 \times 10^{6}\right)$ pre-treated with or without indicated concentrations of different inhibitors were infected with $M$. fortuitum for the indicated time p.i. and were loaded with $\mathrm{DiOC}_{6}(100 \mathrm{nM})$ during the last 30 min of infection then lysed in deionized water, and the reduction in the accumulation of $\mathrm{DiOC}_{6}$ was read in a fluorimeter (HT synergy) at excitation and emission wavelengths of 488 and $500 \mathrm{~nm}$ respectively. The relative change in fluorescence was plotted.

In parallel study, HKM $\left(2 \times 10^{6}\right)$ pre-treated with or without indicated concentrations of different inhibitors were infected with or without $M$. fortuitum for indicated time periods. The cell pellet was harvested, washed with phosphate buffered saline and loaded with JC-1 $(20 \mu \mathrm{M}$, Cayman) for $20 \mathrm{~min}$. The cells were washed, mounted in microslide with cover slips using fluoroshield and the red/ green fluorescence was digitized at indicated time p.i. using confocal microscope ( $\times 40$ oil immersions, $1.30 \mathrm{NA}$, Nikon Eclipse A1Rsi-TiE-300).

\section{Transmission electron microscopy}

HKM $\left(2 \times 10^{7}\right)$ uninfected or infected with M. fortuitum for the indicated time period were washed and fixed with $2.5 \%$ glutaraldehyde (Polaron, Biorad) in $0.1 \mathrm{M}$ phosphate buffer ( $\mathrm{pH}$ 7.4). The fixed HKM were processed as reported earlier ${ }^{24}$ and examined under Tecnai 12 Bio-twin transmission electron microscope (FEI, $80 \mathrm{kV})$.

\section{Calpain assay}

Calpain activity was studied using a fluorogenic activity assay kit (Calbiochem). Briefly, HKM $\left(1 \times 10^{5}\right)$ pre-treated with or without targeted or scrambled siRNAs and specific inhibitors were infected with or without $M$. fortuitum. The HKM were washed at indicated time p.i., lysed, calpain activity measured at $360 \mathrm{~nm}$ excitation and $460 \mathrm{~nm}$ emission respectively and the relative calpain activity plotted.

\section{Caspase assay (caspase-12, caspase-9 and caspase-3)}

Caspase-12, caspase-9 and Caspase-3 were studied using specific assay kits according to the instructions of the manufacturer (Biovision) and using the reagents supplied with the kit. Briefly, HKM $\left(1 \times 10^{6}\right)$ pre-treated with or without indicated concentrations of different inhibitors or targeted or scrambled siRNAs were infected with or without $M$. fortuitum for $24 \mathrm{~h}$. The cells were washed and FITC-tagged caspase-12 inhibitor Z-ATADFMK was added into each culture and left for 30 mins at $30^{\circ} \mathrm{C}$ followed by washing in fluorescein wash buffer. The HKM were mounted on microslides with cover slips using fluoroshield and analyzed under confocal microscope ( $x$ 40 oil immersion $)^{41}$.

For caspase- 9 and caspase- 3 assays the HKM were washed, re-suspended in $50 \mu \mathrm{l}$ of lysis buffer and 
incubated on ice for $10 \mathrm{~min}$. The cell lysate was collected by centrifugation at $10,000 \times g$ for $5 \mathrm{~min}$ at $4{ }^{\circ} \mathrm{C}$. To $50 \mu \mathrm{l}$ of cell lysate, $50 \mu \mathrm{l}$ of $2 \times$ reaction buffer containing DTT $(10 \mathrm{mM})$, PMSF $(5 \mathrm{mM})$ and specific substrates were added, the mixture was incubated at $37^{\circ} \mathrm{C}$ for $5 \mathrm{~h}$ and the absorbance read at $405 \mathrm{~nm}$ in a micro-plate reader (HT synergy $)^{40}$.

\section{Statistical analysis}

Mean \pm SE were calculated for each parameter considered in the present study in the different groups of fish. Pair wise comparison was done by employing $t$-test: two samples using unequal variance to determine the statistical significance between the groups. The value of $p<$ 0.05 was considered statistically significant.

\section{Acknowledgements}

This work was supported by NFBSFARA/ICAR Project Grant (RNAi-2014), University of Delhi Doctoral Research Programme (Dean (R)/R\&D/2012/917) and UGC (MRP-MAJOR-ZOOL-2013-36692). DD, PK, AS, and GV were supported by ICAR Fellowship (Govt of India), CSIR Fellowship (Govt of India), UGC Fellowship (Govt of India), CSIR Fellowship (Govt of India) respectively. We thank A.K. Pal for maintenance of fish.

\section{Author details}

'Department of Zoology, Immunobiology Laboratory, University of Delhi, Delhi, India. ${ }^{2}$ Department of Zoology, Gut Biology Laboratory, University of Delhi, Delhi, India. ${ }^{3}$ Microscopy Laboratory, National Institute of Cholera and Enteric Diseases, Kolkata, India. ${ }^{4}$ Gene Regulation Laboratory, School of Biotechnology, Jawaharlal Nehru University, New Delhi, India

\section{Conflict of interest}

The authors declare that they have no conflict of interest.

\section{Publisher's note}

Springer Nature remains neutral with regard to jurisdictional claims in published maps and institutional affiliations.

Supplementary Information accompanies this paper at https://doi.org/ 10.1038/s41420-018-0034-9.

Received: 27 December 2017 Revised: 18 January 2018 Accepted: 21 January 2018

Published online: 20 February 2018

\section{References}

1. Talaat, A. M., Trucksis, M., Kane, A. S. \& Reimschuessel, R. Pathogenicity of Mycobacterium fortuitum and Mycobacterium smegmatis to goldfish, Carassius auratus. Vet. Microbiol. 66, 151-164 (1999).

2. Bercovier, H. \& Vincent, V. Mycobacterial infections in domestic and wild animals due to Mycobacterium marinum, M. fortuitum, M. chelonae, M. porcinum, M. farcinogenes, M. smegmatis, M. scrofulaceum, M. xenopi, M. kansasii, M. simiae and M. genavense. Rev. Sci. Tech. Int. Epiz. 20, 265-290 (2001).

3. Smith, M. B., Schnadig, V. J., Boyars, M. C. \& Woods, G. L. Clinical and pathologic features of Mycobacterium fortuitum infections an emerging pathogen in patients with AIDS. Am. J. Clin. Pathol. 116, 225-232 (2001).

4. Rojas, M., García, L. F., Nigou, J., Puzo, G. \& Olivier, M. Mannosylated lipoarabinomannan antagonizes Mycobacterium tuberculosis-induced macrophage apoptosis by altering $\mathrm{Ca}^{+}{ }^{2}$-dependent cell signaling. J. Infect. Dis. 182, 240-251 (2000).

5. Mehto, S. et al. Mycobacterium tuberculosis and human immunodeficiency virus type 1 cooperatively modulate macrophage apoptosis via toll like Receptor 2 and calcium homeostasis. PLoS ONE 10, e0131767 (2015).
6. Orrenius, S., Zhivotovsky, B. \& Nicotera, P. Regulation of cell death: the calciumapoptosis link. Nat. Rev. Mol. Cell Biol. 4, 552-565 (2003).

7. Rutkowski, D. T. \& Kaufman, R. J. A trip to the ER: coping with stress. Trends Cell Biol. 14, 20-28 (2004)

8. Marciniak, S. J. et al. CHOPinduces death by promoting protein synthesis and oxidation in the stressed endoplasmic reticulum. Genes Dev. 18, 3066-3077 (2004).

9. Seimon, T. A. et al. Induction of ER stress in macrophages of tuberculosis granulomas. PLOS ONE 5, e12772 (2010)

10. Choi, J. A. et al. Mycobacterial HBHA induces endoplasmic reticulum stressmediated apoptosis through the generation of reactive oxygen species and cytosolic Ca2+in murine macrophage RAW 264.7 cells. Cell Death Dis. 4, e957 (2013).

11. Lim, Y. J. et al. Mycobacterium kansasii-induced death of murine macrophages involves endoplasmic reticulum stress responses mediated by reactive oxygen species generation or calpain activation. Apoptosis 18, 150-159 (2013).

12. Cui, Y. et al. Mycobacterium bovis induces endoplasmic reticulum stress mediated-apoptosis by activating IRF3 in a murine macrophage cell line. Front. Cell Infect. Microbiol. 6, 182, https://doi.org/10.3389/fcimb.2016.00182 (2016).

13. Lim, Y. J. et al. Roles of endoplasmic reticulum stress-mediated apoptosis in M1-polarized macrophages during mycobacterial infections. Sci. Rep. 6, 37211 (2016).

14. Jo, S. H. et al. Calreticulin modulates the intracellular survival of mycobacteria by regulating ER-stress-mediated apoptosis. Oncotarget 8, 58686-58698 (2017).

15. Clapham, D. E. Calcium signaling. Cell 131, 1047-1058 (2007)

16. Hajnóczky, G. et al. Mitochondrial calcium signalling and cell death: approaches for assessing the role of mitochondrial $\mathrm{Ca}^{2+}$ uptake in apoptosis. Cell $\mathrm{Cal}$. 40, 553-560 (2006).

17. Pinton, P., Giorgi, C., Siviero, R., Zecchini, E. \& Rizzuto, R. Calcium and apoptosis: ER-mitochondria $\mathrm{Ca}^{2+}$ transfer in the control of apoptosis. Oncogene 27, 6407-6418 (2008)

18. Szegezdi, E., Logue, S. E., Gorman, A. M. \& Samali, A. Mediators of endoplasmic reticulum stress-induced apoptosis. EMBO Rep. 7, 880-885 (2006).

19. Suzuki, K., Hata, S., Kawabata, Y. \& Sorimachi, H. Structure, activation, and biology of calpain. Diabetes 53, S12-S18 (2004).

20. Francis, R. J., Butler, R. E. \& Stewart, G. R. Mycobacterium tuberculosis ESAT-6 is a leukocidin causing Ca2+influx, necrosis and neutrophil extracellular trap formation. Cell Death Dis. 5, e1474 (2014).

21. Nakagawa, T. \& Yuan, J. Cross-talk between two cysteine protease families Activation of caspase-12 by calpain in apoptosis. J. Cell Biol. 150, 887-894 (2000).

22. Martinez, J. A. et al. Calpain and caspase processing of caspase-12 contribute to the ER stress-induced cell death pathway in differentiated PC12 cells. Apoptosis 15, 1480-1493 (2010)

23. Secombes, C. J. \& Fletcher, T. C. The role of phagocytes in the protective mechanisms of fish. Annu. Rev. Fish. Dis. 2, 53-71 (1992).

24. Datta, D. et al. Calcium and superoxide-mediated pathways converge to induce nitric oxide-dependent apoptosis in Mycobacterium fortuituminfected fish macrophages. PLOS ONE 111, e0146554 (2016).

25. Landowski, T. H., Megli, C. J., Nullmeyer, K. D., Lynch, R. M. \& Dorr, R. T. Mitochondrial-mediated disregulation of $\mathrm{Ca}^{2+}$ is a critical determinant of Velcade (PS-341/bortezomib) cytotoxicity in myeloma cell lines. Can. Res. 65, 3828-3836 (2005)

26. Duan, L., Gan, H., Golan, D. E. \& Remold, H. G. Critical role of mitochondrial damage in determining outcome of macrophage infection with Mycobacterium tuberculosis. J. Immunol. 169, 5181-5187 (2002).

27. Chen, M., Gan, H. \& Remold, H. G. A mechanism of virulence: virulent Mycobacterium tuberculosis strain H37Rv, but not attenuated H37Ra, causes significant mitochondrial inner membrane disruption in macrophages leading to necrosis. J. Immunol. 176, 3707-3716 (2006).

28. Ly, J. D., Grubb, D. R. \& Lawen, A. The mitochondrial membrane potential $\left(\Delta \Psi_{\mathrm{m}}\right)$ in apoptosis; an update. Apoptosis 8, 115-128 (2003).

29. Sakamuru, S. et al. Application of a homogenous membrane potential assay to assess mitochondrial function. Physiol. Genom. 44, 495-503 (2012).

30. Rodrigues, C. M. et al. Ursodeoxycholic acid prevents cytochrome c release in apoptosis by inhibiting mitochondrial membrane depolarization and channel formation. Cell Death Differ. 6, 842-854 (1999).

31. Wu, J. \& Kaufman, R. J. From acute ER stress to physiological roles of the unfolded protein response. Cell Death Differ. 13, 374-384 (2006) 
32. Rao, R. V., Ellerby, H. M. \& Bredesen, D. E. Coupling endoplasmic reticulum stress to the cell death program. Cell Death Differ. 11, 372-380 (2004).

33. Carlisle, R. E. et al. 4 Phenylbutyrate inhibits tunicamycin-induced acute kidney injury via CHOP/GADD153 repression. PLoS ONE 9, e84663 (2014).

34. Kubota, K. et al. Suppressive effects of 4-phenylbutyrate on the aggregation of Pael receptors and endoplasmic reticulum stress. J. Neurochem. 97, 1259-1268 (2006).

35. Iurlaro, R. \& Munoz-Pinedo, C. Cell death induced by endoplasmic reticulum stress. FEBS J. 283, 2640-2652 (2016).

36. Marciniak, S. J. et al. CHOP induces death by promoting protein synthesis and oxidation in the stressed endoplasmic reticulum. Genes Dev. 18, 3066-3077 (2004).

37. Goll, D. E., Thompson, V. F., Li, H., Wei, W. \& Cong, J. The calpain system. Physiol. Rev. 83, 731-801 (2003).

38. Arthur, J. S. C., Elce, J. S., Hegadorn, C., Williams, K. \& Greer, P. A. Disruption of the murine calpain small subunit gene, Capn4: calpain is essential for embryonic development but not for cell growth and division. Mol. Cell Biol. 20, 4474-4481 (2000)

39. Lepage, S. E. \& Bruce, A. E. Characterization and comparative expression of zebrafish calpain system genes during early development. Dev. Dyn. 237, 819-829 (2008).

40. Banerjee, C., Goswami, R., Verma, G., Datta, M. \& Mazumder, S. Aeromonas hydrophila induced headkidney macrophage apoptosis in Clarias batrachus involves the activation of calpain and is caspase-3 mediated. Dev. Comp. Immunol. 37, 323-333 (2012).

41. Banerjee, C. et al. Ameliorating ER-stress attenuates Aeromonas hydrophila induced mitochondrial dysfunctioning and caspase mediated HKM apoptosis in Clarias batrachus. Sci. Rep. 4, 5820 (2014).

42. Morishima, N., Nakanishi, K., Takenouchi, H., Shibata, T. \& Yasuhiko, Y. An endoplasmic reticulum stress-specific caspase cascade in apoptosis cytochrome c-independent activation of caspase-9 by caspase-12. J. Biol. C Hem. 277, 34287-34294 (2002).
43. Sánchez, A., Espinosa, P., García, T. \& Mancilla, R. The 19 kDa Mycobacterium tuberculosis lipoprotein ( $\mathrm{LpqH})$ induces macrophage apoptosis through extrinsic and intrinsic pathways: a role for the mitochondrial apoptosisinducing factor. Clin Dev Immunol. https://doi.org/10.1155/2012/950503 (2012).

44. Cui, Y. et al. Mycobacterium bovis induces endoplasmic reticulum stress mediated-apoptosis by activating IRF3 in a murine acrophage cell line. Front Cell Infect. Microbiol.6, 182 (2016).

45. Lee, K. I. et al. Mycobacterium avium MAV2054 protein induces macrophage apoptosis by targeting mitochondria and reduces intracellular bacterial growth. Sci. Rep. 6, 37804 (2016).

46. Kasahara, K. et al. Expression of chemokines and induction of rapid cell death in human blood neutrophils by Mycobacterium tuberculosis. J. Infect. Dis. 178, 127-137 (1998).

47. Kelly, D. M., ten Bokum, A. M. C., O'Leary, S. M., O'Sullivan, M. P. \& Keane, J. Bystander macrophage apoptosis after Mycobacterium tuberculosisH37Ra infection. Infect. Immun. 76, 351-360 (2008).

48. Rachel, E. et al. The balance of apoptotic and necrotic cell death in Mycobacterium tuberculosis infected macrophages is not dependent on bacterial virulence. PLOS ONE 7, e47573 (2012)

49. Obeng, E. A. \& Boise, L. H. Caspase-12 and caspase-4 are not required for caspase-dependent endoplasmic reticulum stress-induced apoptosis. J. Biol. Chem. 280, 29578-29587 (2005).

50. Boehning, D. et al. Cytochrome $\mathrm{c}$ binds to inositol $(1,4,5)$ trisphosphate receptors, amplifying calcium-dependent apoptosis. Nat. Cell Biol. 5, 1051-1061 (2003)

51. Roca, F. J. \& Ramakrishnan, L. TNF dually mediates resistance and susceptibility to mycobacteria via mitochondrial reactive oxygen species. Cell 153, 521-534 (2013).

52. Banerjee, C. et al. Role of Calmodulin-Calmodulin Kinase II, CAMP/Protein Kinase A and ERK 1/2 on Aeromonas hydrophila-induced apoptosis of headkidney macrophages. PLoS Pathog. 10, e1004018 (2014). 